\title{
Necrotizing Enterocolitis Symposium: Epidemiology and Early Diagnosis
}

\author{
Simon Eaton \\ UCL Great Ormond Street Institute of Child Health and Great Ormond Street Hospital \\ for Children, London
}

\author{
Corresponding author: \\ Simon Eaton, $\mathrm{PhD}$ \\ Department of Paediatric Surgery, \\ UCL Great Ormond Street Institute of Child Health, \\ 30 Guilford Street, \\ London, \\ WC1N 1EH \\ UK \\ Telephone: +44 (0)20 79052158 \\ Fax: +44 (0)20 74046181 \\ Email: s.eaton@ucl.ac.uk
}

Keywords: necrotizing enterocolitis, 


\begin{abstract}
Despite decades of research on necrotizing enterocolitis (NEC), the outlook for infants afflicted by this devastating disease is all too often bleak. The aim of this symposium at the BAPS conference in Amsterdam was to highlight recent advances in the knowledge of the epidemiology and diagnosis of NEC. There were important contributions on the disease in the United Kingdom, both from a neonatal and a surgical point of view, whereas colleagues from the Netherlands and elsewhere in Europe shared their insights into novel diagnostic tools, both biofluid and imaging based.
\end{abstract}


Necrotizing enterocolitis needs no introduction to the paediatric surgeon. The presentation of a sick premature neonate who has been tolerating enteral feeds but has developed abdominal distension, increased aspirates and has pneumatosis intestinalis and pneumoperitoneum is all too familiar, as are the findings at laparotomy - necrotic bowel requiring extensive resection, and the outcomes - mortality, neurodevelopmental impairment and short bowel syndrome [1]. Probiotics have not eradicated the disease [2] and the surgical outcomes do not appear to have improved over twenty years [3, 4], so research into the epidemiology and rapid diagnosis of the disease is urgently required. Although animal models have been used and novel therapies suggested in these models, careful clinical research continues to deliver important data which can hopefully help paediatric surgeons to improve outcomes. The aim of the Symposium at the BAPS Congress in Amsterdam was to provide the paediatric surgeons faced with this disease up-to date information about the epidemiology of NEC across the UK, and novel data on biomarkers and physiological measurements that colleagues in Europe are using to shed light on how intervention might be made in a more timely manner in the future.

\section{Epidemiology}

Although comprehensive data on NEC incidence and/or surgical outcomes is available from some counties, areas, or healthcare systems, including population-based data from Sweden [5] and the Vermont-Oxford Network Hull VON [6], data from the UK has been lacking, except for a survey study of 158 NICUs [7]. The UK National Confidential Enquiry into Patient Outcome and Death report on paediatric surgery in 2011 highlighted the gaps in our knowledge about incidence, epidemiology and outcomes of NEC in the UK [8], and following this two national prospective studies were initiated to fill some of these gaps. The UK Neonatal NEC Study, slightly misleadingly, covered England only, but prospectively acquired data from all from all 163 neonatal units between December 2011 and September 2014 and will provide an important neonatal perspective on risk factors and antecedent feeding practices. Professor Neena Modi, from Imperial College London, gave a sneak preview into these as yet unpublished data, including the development of a consistent case-definition tool for surveillance, and we look forward to the publication of these important data. The other UK study presented at the meeting, the

British Association of Paediatric Surgeons Congenital Anomalies Surveillance System 
(BAPS-CASS) Surgical NEC study, did indeed cover the whole of the UK and Ireland by collecting data from 28 neonatal surgical centres over one year. This study complements the neonatal study by providing data on surgical management and outcomes of those infants that required surgery. Miss Kokila Lakhoo presented the data from this study on behalf of BAPS-CASS, which showed that 236 infants required surgery in the UK over one year, and highlighted the variation in surgical practices. In part, this variation is due to the heterogeneity of disease in terms of presentation and area of intestine affected, but also is a result of the lack of evidence for the optimal surgical management. |We also eagerly look forward to publication of the BAPS-CASS study. In both epidemiological studies, infants were identified who died without having surgery. In addition, there are those infants who have a laparotomy in which the whole intestine is necrotic prompting a decision to withdraw active care. This then leads to the question of whether paediatric surgeons should be intervening earlier, and if so, what should be the indications for surgery? This area of clinical research, pursued actively by our Dutch colleagues, formed the second part of the NEC symposium.

\section{Early diagnosis}

Abdominal x-ray has long been the mainstay of diagnosis of definite NEC (pneumatosis intestinalis) and provides the only universally accepted indication for operation (pneumoperitoneum). Blood work in current routine practice is limited to non-specific markers of systemic disease (C-reactive protein, platelets, white-blood cells) that are more indicative to neonatal intensivists to guide medical than useful as an indication to paediatric surgeons on when to operate. Ernst Van Heurn, based in Amsterdam in collaboration with Joep Derikx, presented data on intestinal fatty acid binding protein (IFABP, measured in urine), calprotectin (measured in faeces) and serum amyloid A (SAA, measured in urine) in early diagnosis of NEC and attempted to answer the questions: (i) can these markers be used for early diagnosis?; (ii) can they predict severity of disease?; and (iii) can they be used to guide the re-introduction of enteral feeds? Ernst showed us that the combination of I-FABP and calprotectin gave the highest accuracy in the diagnosis of NEC [9], that SAA together with platelets were the most predictive of surgical NEC [10] and that I-FABP can be useful to direct re-feeding following surgery [11]. However, use of these markers is only useful if the turnaround time by hospital 
laboratories is fast enough for them to be used in clinical decision making and it remains to be seen whether these markers will enter widespread clinical practice. Circulating cytokines are markers of systemic immune status, and pro-inflammatory cytokines have long been known to be elevated in NEC. There has been limited information, however, on whether cytokine measurements can be useful in diagnosis or risk assessment in NEC [12], and this was the topic of the presentation by Thomas Benkoe, from Vienna. Using a cytokine array to measure pro-inflammatory (IL-1b, IL-2,IL-5,IL-6, IL-8, IL-12, IFNgamma, TNF-alpha, TNF-beta) and anti-inflammatory cytokines (IL-4, IL-10), only IL10 ,IL-8 and IL-6 were shown to be significantly higher in surgical NEC than in matched controls [13], similar findings on IL-6 and IL-8 being reported by others [14] [15]. Thomas then focussed in on IL-8, which is a chemoattractant for neutrophils and macrophages whose intestinal expression has been linked with NEC [16], and showed that IL-8 is a suitable marker for preoperative risk assessment, that IL-8 levels obtained within 6 hours prior to surgery correlate with the actual intraoperative disease extent [17] and that high levels correlate with 60-day mortality [18]. Interestingly, although IL-8 is in theory non-specific, whereas I-FABP discussed above is specific for the gut, IL-8 may in fact be a better marker than I-FABP [19]. In Thomas' hospital, and potentially many others, IL- 8 is available as a routine test, so unlike many laboratory-based biomarkers, might prove to be clinically useful, although precise cutoffs and criteria for intervention must be developed. Of course, imaging methodologies have the potential advantage of either real-time information, or at least a faster reporting time than measurement of biomarkers in body fluids, at least until one of these biomarkers is adopted for a point-ofcare test. Jan Hulscher, from Groningen, has been exploring the use of near infra-red spectroscopy (NIRS) in NEC, and shared his data in his symposium talk. NIRS provides a non-invasive measurement of tissue oxygen saturation, which potentially allows a realtime window into intestinal perfusion, which is measured as tissue fractional oxygen saturation after taking into account arterial saturation concomitantly measured by pulse oximetry. Although one might assume that in NEC infants, intestinal perfusion would be lower in infants predisposed to NEC, in fact Jan showed that cerebral oxygenation, rather than intestinal oxygenation, is impaired in the first days of life in infants that go on to get NEC, whereas intestinal perfusion is impaired immediately preceding clinical NEC [20]. 
In a further study, although NIRS could not distinguish between definite NEC and absence of NEC, it could differentiate between complicated from uncomplicated NEC [21]. Encouragingly, these differences in NIRS readings also correlated well with IFABP levels, thus validating the use of NIRS to examine intestinal impairment [22]. However, Jan stressed that at the present time, because of the high variability, NIRS was currently useful to examine the pathogenesis of NEC at a group level, but that it was not currently ready for prime-time on an individual patient basis, in other words the holy grail of indicating which infants require urgent surgical intervention.

\section{Acknowledgements}

SE gratefully acknowledges support from Great Ormond Street Hospital Children's Charity. Thomas Benkoe, Jan Hulscher, Ernst Van Heurn, Joep Derikx, Kokila Lakhoo, Cheryl Battersby and Neena Modi are all thanked for their contributions to the symposium and their willingness to share their slides to aid my memory in writing this.

\section{References}

1. Hall NJ, Eaton S, Pierro A: Necrotizing enterocolitis: Prevention, treatment, and outcome. Journal of Pediatric Surgery 48:2359-2367, 2013

2. Fleming P, Hall NJ, Eaton S: Probiotics and necrotizing enterocolitis. Pediatric Surgery International 31:1111-1118, 2015

3. Fitzgibbons SC, Ching Y, Yu D, et al: Mortality of necrotizing enterocolitis expressed by birth weight categories. J Pediatr Surg 44:1072-1075, 2009

4. $\quad$ Thyoka M, De Coppi P, Eaton S, et al: Advanced necrotizing enterocolitis part 1: mortality. EurJ PediatrSurg 22:8-12, 2012

5. Ahle M, Drott P, Andersson RE: Epidemiology and Trends of Necrotizing Enterocolitis in Sweden: 1987â€"'2009. Pediatrics 132:e443-e451, 2013

6. Hull MA, Fisher JG, Gutierrez IM, et al: Mortality and management of surgical necrotizing enterocolitis in very low birth weight neonates: a prospective cohort study. J Am Coll Surg 218:1148-1155, 2014

7. Rees CM, Eaton S, Pierro A: National prospective surveillance study of necrotizing enterocolitis in neonatal intensive care units. Journal of Pediatric Surgery 45:1391-1397, 2010

8. Mason DG, Puntis JWL, McCormick K, et al: Parenteral nutrition for neonates and children: a mixed bag. Archives of Disease in Childhood 96:209-210, 2011 
9. Reisinger KW, Van der Zee DC, Brouwers HA, et al: Noninvasive measurement of fecal calprotectin and serum amyloid A combined with intestinal fatty acid-binding protein in necrotizing enterocolitis. J Pediatr Surg 47:1640-1645, 2012

10. Reisinger KW, Kramer BW, Van der Zee DC, et al: Non-invasive serum amyloid A (SAA) measurement and plasma platelets for accurate prediction of surgical intervention in severe necrotizing enterocolitis (NEC). PLoS One 9:e90834, 2014

11. Reisinger KW, Derikx JP, Thuijls G, et al: Noninvasive measurement of intestinal epithelial damage at time of refeeding can predict clinical outcome after necrotizing enterocolitis. Pediatr Res 73:209-213, 2013

12. Evennett NJ, Alexander N, Petrov M, et al: A systematic review of serologic tests in the diagnosis of necrotizing enterocolitis. J Pediatr Surg 44:2192-2201, 2009

13. Benkoe $\mathrm{T}$, Baumann $\mathrm{S}$, Weninger $\mathrm{M}$, et al: Comprehensive evaluation of 11 cytokines in premature infants with surgical necrotizing enterocolitis. PLoS One 8:e58720, 2013

14. Maheshwari A, Schelonka RL, Dimmitt RA, et al: Cytokines associated with necrotizing enterocolitis in extremely-low-birth-weight infants. Pediatr Res 76:100-108, 2014

15. Bhatia AM, Stoll BJ, Cismowski MJ, et al: Cytokine levels in the preterm infant with neonatal intestinal injury. Am J Perinatol 31:489-496, 2014

16. Nanthakumar NN, Fusunyan RD, Sanderson I, et al: Inflammation in the developing human intestine: A possible pathophysiologic contribution to necrotizing enterocolitis. ProcNatlAcadSciUS A 97:6043-6048, 2000

17. Benkoe T, Reck C, Gleiss A, et al: Interleukin 8 correlates with intestinal involvement in surgically treated infants with necrotizing enterocolitis. J Pediatr Surg 47:1548-1554, 2012

18. Benkoe $\mathrm{T}$, Reck C, Pones M, et al: Interleukin-8 predicts 60 -day mortality in premature infants with necrotizing enterocolitis. J Pediatr Surg 49:385-389, 2014

19. Benkoe TM, Mechtler TP, Weninger M, et al: Serum levels of interleukin-8 and gut-associated biomarkers in diagnosing necrotizing enterocolitis in preterm infants. J Pediatr Surg 49:1446-1451, 2014

20. Schat TE, van der Laan ME, Schurink M, et al: Assessing cerebrovascular autoregulation in infants with necrotizing enterocolitis using near-infrared spectroscopy. Pediatr Res, 2015

21. Schat TE, Schurink M, van der Laan ME, et al: Near-Infrared Spectroscopy to Predict the Course of Necrotizing Enterocolitis. PLoS One 11:e0154710, 2016

22. Schat TE, Heida FH, Schurink M, et al: The relation between splanchnic ischaemia and intestinal damage in necrotising enterocolitis. Arch Dis Child Fetal Neonatal Ed, 2016 